\title{
乡村旅游政策变迁影响路径的组态视角研究 基于黄山案例地的定性比较分析
}

\author{
张圆刚 ${ }^{1}$, 黄业坚 $^{2^{*}}$, 余向洋 $^{3}$ \\ (1. 上海师范大学旅游学院, 上海 200234; 2. 中山大学旅游学院, 广州 510275 ; \\ 3. 黄山学院旅游学院, 安徽黄山 245041)
}

\begin{abstract}
摘 要: 在中国制度背景下,乡村旅游的发展与政策变迁紧密相关。论文采用多变量交互作用的清晰集定性比较分 析(csQCA)方法,基于多源流理论探索乡村旅游政策变迁的动力机制, 分析由组织/领导变更、乡村规划政策变更、 本地文化政策变更、东道主居民参与以及旅游市场與情组成的乡村旅游政策变迁影响的构型。研究发现: (1) 单项 因素影响中,组织/领导的变更对文旅局层面的旅游政策出台产生了更大的影响力,东道主居民参与在市级政策的 出台上产生了更大的影响力,但所有单因素均不构成乡村旅游政策变迁的必要条件; (2) 在可能形成的 32 条路径组 合中,存在 4 个充分性条件的特定组合路径影响市级层面的乡村旅游政策变迁, 3 个充分性条件特定组合路径影响 文旅局层面的乡村旅游政策变迁; (3) 对于市级旅游政策变迁来说,乡村规划政策变更、旅游市场與情和东道主居 民参与具有同等重要的影响力, 旅游部门的领导变更在组合中影响力相对较小; (4) 对于文旅局层面的政策变更来 说, 组织领导变更、乡村规划政策变更、本地文化政策变更具有同等重要的影响力,东道主居民参与和旅游市场與 情在路径中影响较小; (5) 多源流的政策变迁动力下旅游政策的效应涉及社会民生等多领域。研究可为未来政策 研究领域的深人与政策制定提供参照。
\end{abstract}

关 键 词:乡村旅游政策; 政策变迁; 影响路径; 定性比较分析; 黄山

乡村旅游是中国解决三农问题与实现乡村振 兴的重要手段与有效途径 ${ }^{[1]}$ 。在中国制度背景下, 乡村旅游的高质量发展有赖于科学合理的政策体 系与制度框架[2]。中国乡村旅游政策也在近几十年 内不断变迁以适应时代变化。这一过程中, 政策地 位逐渐提高 ${ }^{[3}$, 政策发文主体层级逐步上升, 发文部 门日趋多元, 但有研究指出在政策变迁中存在顾此 失彼的“政策洞”等问题 $[4]$ 。因此,对乡村旅游政策 变迁的动力机制进行分析是把握乡村旅游发展规 律、寻找发展动力机制的有效方式, 对政策文件进
行质性与量化研究可以最大程度地诠释政策实践 过程 ${ }^{[5]}$ 。不过, 以往政策变迁分析多以解读政策发 展历史进程为主要内容, 在当下乡村旅游利益主体 日益多元化的过程中, 需要对政策变迁的多元动力 进行探索。不同要素如何单独或组合影响乡村旅 游的政策更新,不同组合如何在政策变迁中产生动 力? 这些问题的解答能够为政策变迁提供新的视 角。因此,本文以黄山乡村旅游政策变迁为研究对 象, 运用定性比较分析 (qualitative comparative analysis, QCA)探索影响乡村旅游政策变迁的要素及其

收稿日期: 2020-05-13; 修订日期 : 2020-07-11

基金项目: 国家自然科学基金项目(41571140); 上海师范大学旅游学院学术前瞻性研究项目(KY2020-BX4)。[Foundation: National Natural Science Foundation of China, No. 41571140; Academic Prospective Rresearch Project of College of Tourism, Shanghai Normal University, No. KY2020-BX4. ]

第一作者简介: 张圆刚(1982-), 男,安徽黄山人,博士, 副教授,主要研究方向为乡村旅游、旅游经济。

E-mail: 634985206@qq.com

*通信作者简介: 黄业坚(1973-), 男, 澳门人,博士,教授,博士生导师, 研究方向为旅游者行为、旅游经济。

E-mail: anthonywip@gmail.com

引用格式: 张圆刚, 黄业坚, 余向洋.乡村旅游政策变迁影响路径的组态视角研究: 基于黄山案例地的定性比较分析 [J]. 地理科学进展, 2021, 40(3): 457-467. [Zhang Yuangang, Wong Ipkin Anthony, Yu Xiangyang. Pathway of influence of rural tourism policy change from the perspective of configuration: Qualitative comparative analysis based on Huangshan case study. Progress in Geography, 2021, 40(3): 457467. ] DOI: 10.18306/dlkxjz.2021.03.009 
组合路径, 以期为未来政策研究领域的深人与政策 制定提供参照。

\section{1 理论基础}

\section{1 政策变迁动力}

政策变迁是政策研究领域中的一个重要主 题 ${ }^{[6]}$, 通常涉及 3 个主流理论, 包括间断均衡理论 (punctuated equilibrium theory)、倡议联盟理论 (initiative alliance theory) 和多源流理论 (multiple streams theory ${ }^{[7]}$ 。间断均衡理论来自 Jones 等 ${ }^{[8]}$, 源于生物进化的 “间断均衡说” ${ }^{[9]}$, 该理论认为政策 变迁是决策制定系统建立和破除政策均衡结构的 过程 ${ }^{[10]}$ 。中国研究者已将间断均衡理论应用到农 村政策变迁研究 ${ }^{[1-12]}$, 丁琳琳等 ${ }^{[13]}$ 指出, 在农村政策 制定中, 自下而上的多元主义政策创新中会促进政 策均衡的更新。倡议联盟框架由 Sabatier 等 ${ }^{[14-15]}$ 在 20 世纪 80 年代提出,该理论认为政策问题的参与 者,例如专家学者、社会组织、政府部门等共同推动 了政策的变迁 ${ }^{[16-17]}$ 。有学者基于倡议联盟框架分析 了农村低保政策 ${ }^{[18-19]}$; 吴光芸等 ${ }^{[20]}$ 研究指出宏观制 度环境、政治精英理念的更新是农地流转政策变迁 的动力机制。多源流理论由 Kingdon ${ }^{[2]}$ 提出, 认为 政策动力来自 3 大源流的共同作用, 即问题源流、政 策源流和政治源流。其中, 问题源流指焦点事件等 重大问题的出现对政策出台的推动 ${ }^{[22]}$; 政策源流指 利益相关者组成的政策共同体的政策建议; 政治源 流指重要政治活动的发生 ${ }^{[19]}$ 。多源流理论被广泛 应用于中国多行业的政策变迁分析之中 ${ }^{[22]}$ 。在农 村政策研究中, 尹云龙 ${ }^{[23]}$ 基于多源流理论分析了中 国扶贫政策变迁动力。随着旅游业影响因素的日 益复杂, 旅游政策的变迁受到社会、政治、经济及與 论等多要素影响, 多源流理论在解释旅游政策变迁 中显示出巨大潜力。

\section{2 中国乡村旅游政策变迁研究}

国家或地区为促进旅游发展会制定一系列关 于旅游的政策 ${ }^{[24]}$ 。当下, 中国旅游政策研究分析多 从宏观的国家政策整体出发分析其发展历程 ${ }^{[25-26]}$, 或在省级层面分析政策演变 ${ }^{[27-29]}$, 也包括对国外旅 游政策借鉴 ${ }^{[30]}$ 和旅游政策效用研究 ${ }^{[31]}$ 。中国乡村旅 游发展具有明显的政策导向性 ${ }^{[32]}$, 唐晓云 ${ }^{[33]}$ 认为, 中 国旅游政策演化从资源配置的行政化正在转向资 源配置的市场化。日益更新的乡村旅游政策也体
现出中国旅游政策目标的多元化进程,体现出政策 工具由强制工具为主导转向为以互动式影响工具 为主导的过程 ${ }^{[34]}$ 。不过, 研究者也指出了中国农村 政策制定的问题，例如，政策对乡村旅游项目设计 和培育支持不足 ${ }^{[35]}$; 缺失连续性的政策设计, 导致 中国乡村旅游无序发展等问题 ${ }^{[2]}$ 。

因此,乡村旅游发展政策动力的研究非常必 要,但当下研究中该主题研究还比较缺失。在新型 城镇化进程中,乡村旅游发展面临更为复杂的影响 因素, 探索政策动力机制是保证未来能够及时且合 理制定乡村旅游政策的前提。同时,由于影响要素 的复杂, 各要素并不仅仅单独发挥作用, 不同要素 间的组合也会产生不同的动力结果。因此,本文基 于多源流理论运用定性比较分析(QCA)方法, 对上 述实践问题进行实证探索。

\section{2 研究设计与案例选择}

\section{1 研究方法}

定性比较分析(QCA)方法最早由 C. C. Ragin 在 其专著 The comparative method: Moving beyond qualitative and quantitative strategies ${ }^{[36]}$ 中提出, 经过 30 多年的发展,该方法已得到普遍关注和应用。这 种分析方法最初被应用于政治学和管理学领域,并 逐步扩展到其他学科,在处理多样性导向的研究时 具有较强的方法优势, 能够强化理论与数据分析的 关联性 ${ }^{[37]}$ 。本文主要运用清晰集定性比较分析 (csQCA)方法, 该方法强调通过实证数据以及相关 理论的不断对话, 从中、小型样本数据中挖掘出研 究问题可能的因果关系。 csQCA 假定研究问题的 因果关系是非线性的,解释条件对结果的影响是相 互依赖的,并且同一现象的发生可能有不同的条件 组合。csQCA一般用以分析二分赋值(变量赋值分 别为 $0 / 1)$, 在实际操作中必须将条件变量与结果变 量根据案例实际情况与理论知识赋值为 0 或 1 , 根 据布尔代数(Boolean algebra) 原则对条件组合进行 简化 ${ }^{[36-37]}$

本文采用清晰集定性比较分析(csQCA)方法探 索黄山市乡村旅游政策变迁的影响因素,主要考虑 以下几点: 第一, csQCA方法的主要特征是它对二 分变量的处理,尤其针对某个现象“发生”和“未发 生”进行判断, 因此适合于本文中对政策是否有变 迁, 以及其潜在的推动要素是否发生进行判 ${ }^{[}{ }^{[38]}$; 
第二, csQCA方法的核心并不是将各条件变量认为 是独立影响结果变量的因素, 而是将其以不同方式 组合作用于结果变量, 该方法不关注条件变量对结 果变量的净效应, 而是专注于挖掘出能够对结果变 量有解释力的不同要素组合。黄山市乡村旅游政 策变迁的结果同时取决于不同的影响因素,使用 csQCA 方法能挖掘出具有同等重要效应的多条路 径 ${ }^{[39]}$; 第三,引发黄山市乡村旅游政策变迁的单个因 素对结果之间的关系并非对称, 而 csQCA 恰好认为 导致某结果发生与不发生的前因变量具有不对称 性,因此该方法很好地契合了本文主题;第四,使用 csQCA方法可以对同一案例区内不同个案之间进 行比较, 探寻其中符合理论逻辑的前因条件组合, 并 建立各种条件组合与被解释结果的因果关系。

\section{2 乡村旅游政策变迁案例地选择}

发展乡村旅游是实施乡村振兴战略的有效路 径,也是改善农村人居环境的有力举措。近年来, 黄山市借助黄山品牌效应, 通过古徽州特色文化与 乡村的结合, 构建以徽文化为核心、以主题村落为 主要载体的乡村休闲旅游地。黄山市是全国乡村 旅游提升和旅游扶贫大会举办地, 其中黟县成为首 批中国旅游强县, 西递、宏村在 2000 年人选世界文 化遗产名录, 被评为中国十大乡村旅游目的地。黄 山市还拥有 2 个全国休闲农业与乡村旅游示范县, 9 个全国农业旅游示范点。2018 年, 黄山市共接待乡 村旅游游客 4450 万人次, 同比增长 $6.76 \%$; 乡村旅 游总收人 315 亿元, 同比增长 $5.62 \%$ 。2019年 1-6 月, 黄山市乡村旅游接待人数 2413.12 万人次, 同比 增长 $14.75 \%$; 全市乡村旅游总收人 188.03 亿元, 同 比增长 $18.16 \%$ 。选择黄山市乡村旅游政策变迁的 影响因素与组合路径, 具有典型性和代表性。

\section{3 解释变量}

以往研究者指出了乡村旅游政策的若干影响

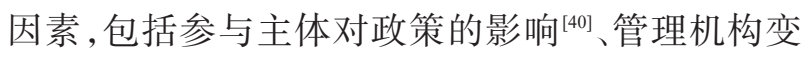
动 ${ }^{[4]}$, 以及市场变动 ${ }^{[27]}$ 对政策制定的影响等。随着 旅游政策影响源流的日益复杂, 需要对以往单要素 分析进行系统整合。基于多源流理论, 可从 3 个源 流层面来分析中国旅游政策变迁动力, 综合乡村旅 游实践与以往研究成果, 可发现政策源流包括: 第 一, 乡村规划政策变动。土地调整是中国农地制度 的一个重要特征 ${ }^{[42]}$,乡村规划政策的变动对旅游产 业发展起着基础性的作用。第二,文化政策的变 动。乡村文化景观保护是中国乡村文化治理研究
的重要内容 ${ }^{[43]}$ 。在乡村旅游发展初期, 经济效应是 乡村开发的首要追求, 而随着文化价值观的变动, 文化政策开始越来越多地影响旅游政策的制定。 在旅游政策变迁中, 问题源流主要反映为旅游市场 與情。在互联网时代, 网络與情事件正在对旅游目 的地形象带来新的威胁, 值得引起学界关注 ${ }^{[4]}$ 。與 情的影响已经成为旅游政策的重要变迁动力, 例如 2015 年青岛大虾事件后, 多部门第一时间联合发布 政策以应对與情。因此,在乡村旅游方面也需要将 该因素考虑进去。旅游政策更新的政治源流包括： 第一,领导层的变动。西方研究者指出, 领导的更 迭是政策变迁的重要因素之一 ${ }^{[7]}$,中国政府部门决 策者的变动也会带来政策的冲击 ${ }^{[1-45]}$ 。第二, 东道 主居民参与。乡村旅游作为有效解决 “三农” 问题 的切人点 ${ }^{[46]}$, 发展乡村旅游的核心目标是提升农村 居民的生活水平与幸福感。随着乡村旅游居民参 与程度的日益提升, 适时调整旅游地管理模式(包 括管理行为与目标)也就成为必然 ${ }^{[39]}$ 。在乡村土地 政策研究中, 易承志 ${ }^{[47]}$ 指出, 一项土地政策的确立 不仅取决于其是否符合国家建设战略的需要,而且 取决于其是否得到农民的支持。因此,东道主居民 的参与是政策出台的重要动力因素。

\section{3 定性比较分析}

\section{1 变量赋值与真值表}

本文在确定条件变量和结果变量之后,依据 $\operatorname{csQCA}$ 分析方法原则需要对设定好的条件变量和 解释变量按照二分类的方式进行赋值 ${ }^{[48]}$ 。数据采 集时间集中于 2020 年 2 月 25 日- 3 月 1 日,政策变 迁案例选择的时间段为近 $10 \mathrm{a}$ 的黄山市乡村旅游 政策变迁,数据来源的方式如下: 黄山市旅游相关 政策的变迁通过对黄山市政府与各部门的官方网 站所公布的政策进行盘点, 同时与黄山市旅游部门 工作人员进行访谈后汇总梳理; 组织领导变更是指 黄山市旅游委员会/黄山市文化和旅游局进行的部 门重组或领导变更; 乡村规划政策变更是指黄山乡 村规划和发展等相关政策的调整,通过对黄山市政 府与黄山市发改委、黄山市农业农村局出台乡村规 划政策进行盘点梳理; 文化政策变动依据的是黄山 市文化类政策的更新,通过对黄山市政府与黄山市 文化和旅游局出台的文化政策进行盘点梳理。在 政策要素的选择中需要指出, 国家和省级层面旅游 
政策的出台必然会引发市级层面政策的响应,将其 纳人任何一个路径都会影响该路径作为整体和路 径中其他要素的判断, 因此在本文中不将其纳人分 析。东道主居民参与是指乡村居民对旅游发展提 出的建议,通过对黄山市政府官方网站中的留言进 行梳理, 篮选出与旅游相关的内容; 旅游市场與情 包括市级、省级和国家级新闻媒体与网络媒体所发 布的黄山旅游负面报道。以上变量的判断方式见 表 1 。在二分法赋值后,构建真值表, 见表 2 。

\section{2 单变量分析}

在 $\operatorname{csQCA}$ 方法分析过程中, 首先通过分析单一 条件变量判断是否为乡村旅游政策制定的充分或必 要条件。其中能构成充分性或必要性条件的解释变 量集合可判断为对应的结果变量集合的子集 ${ }^{[38,49]}$, 其 公式为:

$$
\operatorname{Consistency}\left(X_{i} \leqslant Y_{i}\right)=\frac{\sum \min \left(X_{i}, Y_{i}\right)}{\sum X_{i}}
$$

式中: $X_{i}$ 为条件组合中的隶属分数; $Y_{i}$ 为结果中的 隶属分数; Consistency 为一致性, 取值范围为 $(0,1)$ 。

通常来说, 当一致性大于 0.9 可视为结果的必 要条件 ${ }^{[7,50]}$ 。从分析结果可以看出(表 3 ), 5 个条件变 量都并非构成结果变量的必要条件,在本文中体现 为并未一定导致市级或文旅局层面政策的出现。

进一步, 可通过覆盖率指标(Coverage)来判断 条件 $X$ 对于 $Y$ 的解释力度 ${ }^{[00]}$ 。覆盖率表示条件变量 对于因果路径的解释能力, 覆盖率数值越高, 对于 因果的解释力能力越好, 公式为:

$$
\operatorname{Coverage}\left(X_{i} \leqslant Y_{i}\right)=\frac{\sum \min \left(X_{i}, Y_{i}\right)}{\sum Y_{i}}
$$

式中: Coverage 取值范围为 $(0,1)$ 。

通过表 3 可以看出,对于市级旅游政策来说,乡 村规划政策变更和旅游市场與情是一致性最高的 单变量因素, 反映了乡村发展与市场的力量。对于 文旅局级的政策来说,乡村规划政策的变更和文化

表 1 解释变量和结果变量的设定

\begin{tabular}{|c|c|c|c|c|}
\hline 变量类型 & 变量 & 测量变量 & 判断依据 & 赋值 \\
\hline \multirow[t]{10}{*}{ 条件变量 } & 组织/领导变更 & 旅游相关部门组织变更/领导层更换 & 旅游相关部门组织变更/领导层更换 $\geqslant 1$ 次 & 1 \\
\hline & & & 无 & 0 \\
\hline & 乡村规划政策 & 乡村规划政策变更 & 乡村规划政策更新 $\geqslant 1$ 次 & 1 \\
\hline & & & 无 & 0 \\
\hline & 文化政策变动 & 本地文化政策更新 & 相关文化保护政策出台 $\geqslant 1$ 次 & 1 \\
\hline & & & 无 & 0 \\
\hline & 东道主居民参与 & 市政府网旅游主题留言 & 相关建议出现次数 $\geqslant 1$ 次 & 1 \\
\hline & & & 无 & 0 \\
\hline & 旅游市场與情 & 旅游與情危机发生 & 发生次数 $\geqslant 1$ 次 & 1 \\
\hline & & & 无 & 0 \\
\hline \multirow[t]{2}{*}{ 结果变量 } & 政策变迁 & 市级政策的更新 & 黄山市委/市政府/人大 & 1 \\
\hline & & & 文旅局 & 0 \\
\hline
\end{tabular}

Tab.1 Value assignment of explanatory and result variables

表 2 解释和被解释变量清晰集真值

Tab.2 Clear set truth table of explanatory and explained variables

\begin{tabular}{ccccccc}
\hline 案例序号 & 组织/领导变更 & 乡村规划政策变更 & 文化政策变更 & 东道主居民参与 & 旅游市场舆情 & 旅游政策变迁 \\
\hline 1 & 0 & 1 & 0 & 1 & 0 & 1 \\
2 & 0 & 1 & 1 & 0 & 1 & 1 \\
3 & 1 & 1 & 1 & 1 & 0 & 1 \\
4 & 1 & 0 & 0 & 0 & 1 & 0 \\
5 & 1 & 1 & 1 & 1 & 0 & 0 \\
6 & 0 & 1 & 0 & 1 & 0 & 1 \\
7 & 1 & 0 & 1 & 1 & 0 & 0 \\
8 & 0 & 1 & 1 & 0 & 1 & 0 \\
\hline
\end{tabular}


表 3 单因素充分与必要性分析

Tab.3 Single factor sufficiency and necessity analysis

\begin{tabular}{|c|c|c|c|c|}
\hline \multirow{2}{*}{ 条件变量 } & \multicolumn{2}{|c|}{ 一致性 } & \multicolumn{2}{|c|}{ 覆盖率 } \\
\hline & 市级政策 & 旅游部门政策 & 市级政策 & 旅游部门政策 \\
\hline 组织/领导变更 & 0.400 & 0.500 & 0.500 & 0.500 \\
\hline 乡村规划政策变更 & 0.800 & 0.750 & 0.571 & 0.429 \\
\hline 文化政策变更 & 0.600 & 0.750 & 0.500 & 0.500 \\
\hline 东道主居民参与 & 0.600 & 0.500 & 0.600 & 0.400 \\
\hline 旅游市场舆情 & 0.800 & 0.250 & 0.800 & 0.200 \\
\hline
\end{tabular}

政策的变更对旅游政策的出台起到了最大的推动 作用。从影响要素的角度看, 旅游部门组织/领导的 变更对文旅局层面的旅游政策出台产生了更大的 影响力, 东道主居民参与在市级政策的出台上产生 了更大的影响力。但是,所有单因素都不构成乡村 旅游政策变迁的充要条件, 不同因素需要进行条件 组合发挥作用。

\section{4 多因素组合分析}

通过上述分析可知, 单个条件因素的影响均无 法解释乡村旅游政策变迁的原因, 因此, 需要进行 多因素的组合分析。运用软件 fsQCA 3.0 对案例数 据进行分析, 选择复杂解进行解读。市级政策和
文旅局层面政策的复合方案结果分别如表 4 和表 5 所示。

总体来看, 通过应用清晰集定性比较分析方 法, 可见, 市级层面和文旅局层面原生覆盖率均高 于 (或等于) 0.2 , 说明能够解释 $20 \%$ 及以上的案例; 唯一覆盖率均高于(或等于) 0.2 , 表明 $20 \%$ 及以上案 例能够被该条路径解释(不能用于解释其他路径)。 市级旅游政策的变迁在计算精简后共有 4 组充分条 件组合, 整体覆盖率约为 1 , 整体一致性约为 1 , 均达 到了 0.9 以上的阈值标准。因此,该 4 个条件组合能 够有效解释市级旅游政策变迁的影响因素组合。 在文旅局级别的政策变更中,计算精简后共有 3 组 充分条件组合,整体覆盖率约为 1 , 整体一致性约为 1 ,也均达到了 0.9 以上的阈值标准。因此,该 3 个条

表4 多因素的组合结果(市级政策)

Tab.4 Combination results of multiple factors (municipal policies)

\begin{tabular}{|c|c|c|c|c|}
\hline 序号 & 组合 & 原生覆盖率 & 唯一覆盖率 & 一致性 \\
\hline 1 & ～旅游市场舆情*东道主居民参与* 文化政策变更* 组织/领导变更*乡村规划政策变更 & 0.2 & 0.2 & 1 \\
\hline 2 & 旅游市场與情*东道主居民参与*～文化政策变更*组织/领导变更* 乡村规划政策变更 & 0.4 & 0.4 & 1 \\
\hline 3 & 旅游市场舆情* 东道主居民参与*文化政策变更* 组织/领导变更*乡村规划政策变更 & 0.2 & 0.2 & 1 \\
\hline 4 & 旅游市场舆情*东道主居民参与*文化政策变更*组织/领导变更*乡村规划政策变更 & 0.2 & 0.2 & 1 \\
\hline \multicolumn{5}{|c|}{ 整体覆盖率: 1} \\
\hline \multicolumn{5}{|c|}{ 整体一致性: 1} \\
\hline
\end{tabular}

注:“*”是变量之间的连接符号,表示“且”的交集关系; “ ”表示“非”,即“不存在”; 原生覆盖率表示每条路径能够解释案例的百分比;唯 一覆盖率表示案例能够被该条路径解释的百分比,而不能用于解释其他路径; 整体覆盖率表示所有组合路径解释了结果变量案例的百分比; 3 个覆盖率均采用式(2)进行计算。下同。

表 5 多因素的组合结果(文旅局层面政策)

Tab.5 Combination results of multiple factors (tourism bureau level)

\begin{tabular}{|c|c|c|c|c|}
\hline 序号 & 组合 & 原生覆盖率 & 唯一覆盖率 & 一致性 \\
\hline 1 & ～旅游市场與情* 东道主居民参与*文化政策变更*组织/领导变更* 乡村规划政策变更 & 0.25 & 0.25 & 1 \\
\hline 2 & ～旅游市场與情*东道主居民参与*文化政策变更* 组织/领导变更*乡村规划政策变更 & 0.50 & 0.50 & 1 \\
\hline 3 & 旅游市场與情* 东道主居民参与*文化政策变更*组织/领导变更*乡村规划政策变更 & 0.25 & 0.25 & 1 \\
\hline \multicolumn{5}{|c|}{ 整体覆盖率: 1} \\
\hline \multicolumn{5}{|c|}{ 整体一致性: 1} \\
\hline
\end{tabular}


件组合能够有效解释文旅局级政策变迁的影响因 素组合。乡村旅游政策变迁影响路径模型如图 1 所示。

市级旅游政策的变迁中, 具体来看, 路径 1 显示 为 “ 旅游市场與情*东道主居民参与* 文化政策变 更* 组织/领导变更*乡村规划政策变更”, 该路径 围绕东道主居民参与和乡村规划/政策变更发挥作 用,体现乡村自身推动力。路径 2 显示为“旅游市场 與情*东道主居民参与* 文化政策变更*组织/领导 变更* 乡村规划政策变更”, 该路径围绕旅游市场 與情和旅游部门的组织/领导变更发挥作用, 体现出 旅游产业发展的推动力。路径 3 “旅游市场與情* 东道主居民参与*文化政策变更* 组织/领导变更* 乡村规划政策变更” 和路径 4 “旅游市场與情*东道 主居民参与*文化政策变更*组织/领导变更*乡村规 划政策变更”围绕旅游市场與情、文化政策变更和 乡村规划政策变更发挥作用, 体现出市场力量、乡 村自身推动力、文化力量与旅游管理力量的共同作 用。总体来看, 乡村规划变更、旅游市场與情和东 道主居民参与出现在 3 个路径中, 这 3 个变量的两 两组合出现在 4 个路径组合中; 而组织领导的变更 只出现在 2 个路径组合中。

在文旅局层面的政策变更中, 具体来看, 共有 3 条组合路径。路径 1 显示为“ 旅游市场與情* 东 道主居民参与*文化政策变更*组织/领导变更* 乡
村规划政策变更”,该路径围绕文化政策变更和组 织/领导变更发挥作用; 路径 2“ 旅游市场與情*东 道主居民参与*文化政策变更* 组织/领导变更*乡 村规划政策变更”,该路径围绕东道主居民参与、乡 村规划政策变更和文化政策变更发挥作用,体现乡 村力量; 路径 3 “旅游市场與情* 东道主居民参与 * 文化政策变更*组织/领导变更*乡村规划政策变 更”围绕市场與情、组织/领导变更、乡村规划政策 变更发挥作用,体现出市场力量、乡村自身推动力 和旅游管理力量的共同作用。总体来看, 对于文旅 局级别的政策变更来说,在路径 1 和路径 3 中都出 现了组织/领导变更要素,具有和乡村规划变更、文 化政策变更同样的重要推动力; 并且以上 3 个变量 的两两组合出现 3 个路径组合中。相比之下, 东道 主居民参与和旅游市场與情只分别出现在 1 个路 径组合中。

从以上路径组合可以看出, 就黄山市案例地来 说,组织/领导变更、乡村规划政策变更、本地文化 政策变更、东道主居民参与和旅游市场與情 5 个要 素通过不同组合发挥作用,促进了市级和文旅局层 面的乡村旅游政策变更。乡村规划在 2 个层面都 展示出重要的作用力; 市场與情和东道主参与这 2 类具有“自下而上”性质的要素在市级政策层面具 有重要推动力, 而组织/领导变更则在文旅局级别 的政策变迁中更为重要。

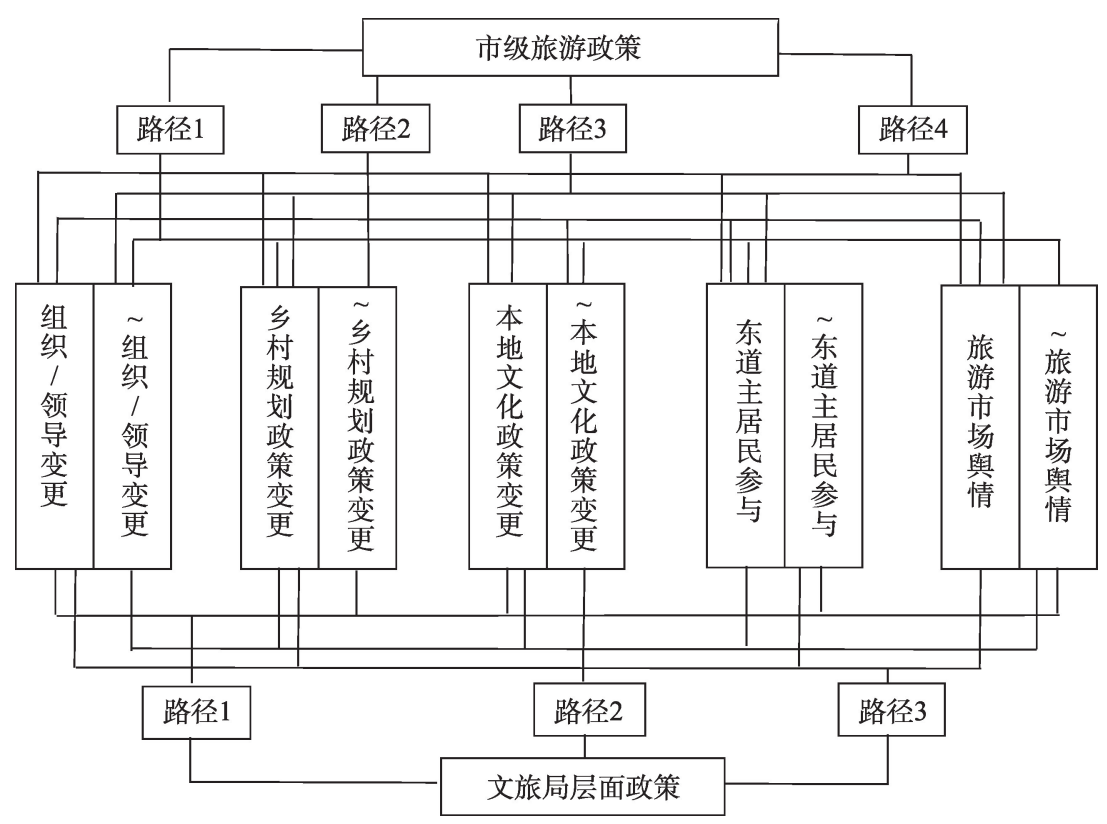

图 1 乡村旅游政策变迁影响路径模型

Fig.1 Influencing factors and path analysis model of rural tourism policy changes 


\section{5 结论与讨论}

本文立足于乡村旅游政策变迁的现实条件,从 乡村旅游政策变迁影响因素由各个单项因素组成 的理论关系出发, 依据数据特点利用清晰集定性比 较分析,探寻由组织/领导变更、乡村规划政策变更、 本地文化政策变更、东道主居民参与以及旅游市场 與情组成的乡村旅游政策变迁影响的构型。该方 法能够更深层次地挖掘乡村旅游政策变迁影响因 素的组合, 这些组合能够很好地解释结果变量。研 究发现:

第一,对于单项因素来说,乡村规划政策变更 和旅游市场與情在市级旅游政策层面一致性相对 较高,乡村规划政策的变更和文化政策的变更在文 旅局层面的政策变迁一致性相对较高,但所有单因 素都不构成乡村旅游政策变迁的必要条件,需要进 行条件组合。

第二,对于市级旅游政策变迁来说, 乡村规划 政策变更、旅游市场與情和东道主居民参与具有同 等重要的影响力,这 3 个变量的两两组合出现在 4 个路径组合之中。相比之下,旅游部门的领导变更 对市级的旅游政策出台影响力相对较小。可以看 出, 市级旅游政策的出台受到更广泛动力影响, 既 会考虑乡村本身的需求,也会对市场與情作出反 应。同时,东道主居民的意见可以指导市级决策层 面,也能够对市级政策的出台起到推动作用。

第三,对于文旅局层面的政策变更来说, 组织 领导变更、乡村规划变更和文化政策变具有同等重 要的影响力, 此 3 个变量的两两组合出现在 3 个路 径组合中。相比之下, 东道主参与和旅游市场與情 只分别出现在 1 个路径组合中。与市级层面的旅游 政策出台相比,文旅局层面的政策更易受到本系统 内组织变更和领导层变动的影响。与此同时,在文 旅融合的背景下, 随着黄山市文化和旅游局的成 立, 文化政策的变更对旅游政策的变迁起到了重要 推动作用。

第四,多因素影响下的旅游相关政策的制定所 产生的政策效果已超越旅游产业边界, 带来了多重 的社会与民生效益。例如在《2018 年黄山市乡村旅 游扶贫实施方案》出台后, 黄山市针对“旅游资源基 础条件好、交通区位条件好、开展乡村旅游扶贫积 极性高” 的 16 个省级旅游扶贫重点村开展游客中 心、停车场、导游词、农家乐和民宿等领域的“八个
一”建设,截至当年底, 实现脱贫 924 户。因此在未 来研究中,可在政策变迁路径分析的基础上,进一 步分析不同路径、不同要素组合,以及不同要素主 导下所实现的旅游政策效果。

\section{参考文献(References)}

[1] 李涛. 中国乡村旅游投资发展过程及其主体特征演化 [J]. 中国农村观察, 2018(4): 132-144. [Li Tao. The development of rural tourism investment and evolution of investors' characteristics. China Rural Survey, 2018(4): 132144. ]

[2] 马静, 舒伯阳. 中国乡村旅游 30 年: 政策取向、反思及优 化 [J]. 现代经济探讨, 2020(4): 116-122. [Ma Jing, Shu Boyang. Rural tourism in China for 30 years: Policy orientation, reflection and optimization. Modern Economic Research, 2020(4): 116-122. ]

[3] 李剑锋, 黄泰圭, 屈学书. 近 30 年来我国乡村旅游政策演 进与前瞻 [J]. 资源开发与市场, 2019, 35(7): 968-972. [Li Jianfeng, Huang Taigui, Qu Xueshu. Rural tourism policy evolution in the past 30 years and future directions in China. Resource Development \& Market, 2019, 35(7): 968-972. ]

[4] 舒伯阳, 马静. 中国乡村旅游政策体系的演进历程及趋 势研究: 基于 30 年数据的实证分析 [J]. 农业经济问题, 2019, 40(11): 94-107. [Shu Boyang, Ma Jing. Evolution and trend of rural tourism policy system in China: An empirical analysis based on 30 years data. Issues in Agricultural Economy, 2019, 40(11): 94-107. ]

[5] 傅雨飞. 公共政策量化分析: 研究范式转换的动因和价 值 [J]. 中国行政管理, 2015(8): 116-120. [Fu Yufei. Quantitative analysis of public policy: Reason and value of the research paradigm transformation. Chinese Public Administration, 2015(8): 116-120. ]

[6] 田华文,魏淑艳. 政策论坛: 未来我国政策变迁的重要动 力: 基于广州市城市生活垃圾治理政策变迁的案例研 究 [J]. 公共管理学报, 2015, 12(1): 24-33, 154. [Tian Huawen, Wei Shuyan. Policy forum: An important impetus in China's future policy changes: Based on the case study on policy changes of living garbage governance in Guangzhou City. Journal of Public Management, 2015, 12 (1): 24-33, 154 . ]

[7] 包国宪, 马翔. 兰州市酒水治污政策变迁路径与动力研 究: 基于定性比较分析法 [J]. 北京理工大学学报(社会 科学版), 2018, 20(4): 29-39. [Bao Guoxian, Ma Xiang. Study on the motivation and path of Lanzhou irrigation pollution control policy: Based on qualitative comparative analysis. Journal of Beijing Institute of Technology 
(Social Sciences Edition), 2018, 20(4): 29-39. ]

[8] Jones B D, Baumgartner F R. From there to here: Punctuated equilibrium to the general punctuation thesis to a theory of government information processing [J]. Policy Studies Journal, 2012, 40(1): 1-20.

[9] 文宏. 间段均衡理论与中国公共政策的演进逻辑: 兰州 出租车政策(1982-2012)的变迁考察 [J]. 公共管理学 报, 2014, 11(2): 70-80, 142. [Wen Hong. The punctuatedequilibrium theory and logic evolution of China's public policy. Journal of Public Management, 2014, 11(2): 7080, 142. ]

[10] 刘开君. 公共政策变迁间断一平衡模型的修正及应用: 兼论新中国科研政策变迁的渐进与突变规律 [J]. 北京 社会科学, 2016(11): 112-120. [Liu Kaijun. Modification and application of public policy's punctuated equilibrium analytical framework: The regularity of gradualism and mutation of scientific research policy change in China. Social Sciences of Beijing, 2016(11): 112-120. ]

[11] 李金龙, 王英伟. “间断平衡框架” 对中国政策过程的解 释力研究: 以 1949 年以来户籍政策变迁为例 [J]. 社会 科学研究, 2018(1): 64-72. [Li Jinlong, Wang Yingwei. A study on the explanatory power of "discontinuous equilibrium framework" to China's policy process. Social Science Research, 2018(1): 64-72. ]

[12] 陈伟, 高力. 间断一均衡模型: 中央 “一号文件”农业政 策变迁的一种分析框架 [J]. 云南行政学院学报, 2015, 17(2): 104-107. [Chen Wei, Gao Li. Discontinuity equilibrium model: An analysis framework of agricultural policy changes in the "No.1 Document" of the central government. The Journal of Yunnan Administration College, 2015, 17(2): 104-107. ]

[13] 丁琳琳, 孟庆国, 刘文勇. 农村集体建设用地人市的发 展实践与政策变迁 $[\mathrm{J}]$. 中国土地科学, 2016, 30(10): 310. [Ding Linlin, Meng Qingguo, Liu Wenyong. Development practice and evolution of policies on rural collective- owned construction land transfer in China. China Land Sciences, 2016, 30(10): 3-10. ]

[14] Sabatier P A. An advocacy coalition framework of policy change and the role of policy-oriented learning therein [J]. Policy Sciences, 1988, 21(2/3): 129-168.

[15] Sabatier P A, Hank C, Smith J. Policy change and learning: An advocacy coalition approach [M]. Boulder, USA: Westview Press, 1993.

[16] 谭爽, 崔佳. 环境 NGO 的政策倡导实践: 基于 “倡议联 盟框架” 的分析 [J]. 南京工业大学学报(社会科学版), 2019, 18(6): 23-32, 111. [Tan Shuang, Cui Jia. Policy ad- vocacy practice of environmental NGOs: Analysis based on the advocacy coalition framework. Journal of Nanjing Tech University (Social Science Edition), 2019, 18(6): 23-32, 111. ]

[17] 桂西. 威权体制下的倡导联盟和政策过程 [D]. 上海: 上 海师范大学, 2013. [Gui Xi. Advocacy alliance and policy process under authoritarian system. Shanghai, China: Shanghai Normal University, 2013. ]

[18] 侯志峰. 政策联盟的注意力配置、互动策略与社会政策 变迁: 基于甘肃农村低保政策的分析个案 [J]. 甘肃行 政学院学报, 2019(2): 45-51. [Hou Zhifeng. Research on the attention distribution, interactive strategy and social policy changing: Based on the advocacy coalition framework. Journal of Gansu Administration Institute, 2019 (2): 45-51.]

[19] 姜艳华, 李兆友. 多源流理论在我国公共政策研究中的 应用述论 [J]. 江苏社会科学, 2019(1): 114-121. [Jiang Yanhua, Li Zhaoyou. On the application of multiplestreams theory in China's public policy research. Jiangsu Social Sciences, 2019(1): 114-121. ]

[20] 吴光芸, 万洋. 中国农村土地流转政策变迁的制度逻 辑: 基于历史制度主义的分析 [J]. 青海社会科学, 2019 (1): 86-94. [Wu Guangyun, Wan Yang. Institutional logic of the policy change of rural land circulation in China: Based on the paradigm of historical institutionalism. Qinghai Social Sciences, 2019(1): 86-94. ]

[21] Kingdon J W. Agendas, alternatives and public policies [M]. Boston, USA: Little, Brown \& Co., 1984.

[22] 甄美荣, 江晓壮, 杨晶照. 基于多源流理论的中国国家 高新区政策变迁动力分析 $[\mathrm{J}]$. 南京工业大学学报(社会 科学版), 2019, 18(6): 88-100, 112. [Zhen Meirong, Jiang Xiaozhuang, Yang Jingzhao. Analysis on motivation of policy changes in China's state high-tech zones: Based on multi-stream framework. Journal of Nanjing Tech University (Social Science Edition), 2019, 18(6): 88-100, 112. ]

[23] 尹云龙. 基于多源流理论视角的我国扶贫政策变迁动 力模式研究 [J]. 学术交流, 2019(1): 126-136. [Yin Yunlong. Research on the motive power model of China's poverty alleviation policy change from the perspective of multiple stream theory. Academic Exchange, 2019(1): 126-136. ]

[24] 韩卢敏, 陆林, 杨兴柱. 安徽省旅游政策变迁及其空间 响应研究 [J]. 地理科学, 2016, 36(3): 431-438. [Han Lumin, Lu Lin, Yang Xingzhu. Change of tourism policy and its spatial response of Anhui Province, China. Scien- 
tia Geographica Sinica, 2016, 36(3): 431-438. ]

[25] 胡北明, 黄俊. 中国旅游发展 70 年的政策演进与展望: 基于 1949-2018 年政策文本的量化分析 [J]. 四川师范 大学学报(社会科学版), 2019, 46(6): 63-72. [Hu Beiming, Huang Jun. Policy evolution and prospects of China's tourism development among 70 years: A quantitative analysis based on policy texts from 1949 to 2018. Journal of Sichuan Normal University (Social Sciences Edition), 2019, 46(6): 63-72. ]

[26] 魏宇, 余青. 基于语义分析的政策差异量化研究: 以近 三十年旅游交通政策为例 [J]. 情报杂志, 2019, 38(3): 194-202. [Wei Yu, Yu Qing. Quantitative research of policy differences based on semantic analysis: Take the tourism transportation policy for the past 3 decades as an example. Journal of Intelligence, 2019, 38(3): 194-202. ]

[27] 刘希否, 梁宏志. 贵州省旅游政策演化的方向、效果及 影响研究 [J]. 经济问题探索, 2009(2): 122-126. [Liu Xilei, Liang Hongzhi. The direction, effects and influences of the tourism policy evolution of Guizhou Province. Inquiry into Economic Issues, 2009(2): 122-126. ]

[28] 刘杰武. 香港回归后粤港地区旅游政策演变研究 [J]. 中国人口・资源与环境, 2013, 23(S1): 154- 157. [Liu Jiewu. Study on the evolution of the tourism policies among Guangdong and Hong Kong after Hong Kong's handover to China. China Population, Resources and Environment, 2013, 23(S1): 154-157. ]

[29] 王芗莹, 严艳. 陕西省旅游政策的递进与绩效研究 [J]. 资源开发与市场, 2013, 29(6): 669-672. [Wang Yingying, Yan Yan. Research on progressive and influence of tourism policies in Shaanxi Province. Resource Development and Market, 2013, 29(6): 669-672. ]

[30] 王慧㜀, 张辉. 中国旅游政策评估模型构建与政策变量 分析 [J]. 旅游科学, 2015, 29(5): 1-13. [Wang Huixian, Zhang Hui. Analysis of the construction of China's tourism policy evaluation model and policy variables. Tourism Science, 2015, 29(5): 1-13. ]

[31] 李锋, 孙根年, 付琦. 基于抗周期性角度的我国旅游产 业政策效用评估研究: 以四次旅游产业政策为例 $[\mathrm{J}]$. 经 济地理, 2013, 33(6): 162-169. [Li Feng, Sun Gennian, $\mathrm{Fu}$ Qi. Study on evaluation of the effectiveness of China's tourism industrial policy based on the view of the periodicity resistance. Economic Geography, 2013, 33(6): 162-169. ]

[32] 周燕. 我国乡村旅游发展的政策回顾与趋势前瞻: 基于 2004 年以来国家层面政策文本分析 [J]. 云南行政学院 学报, 2019, 21(4): 121-124. [Zhou Yan. Policy review and trend forecast of rural tourism development in China: Based on the analysis of policy texts at the national level since 2004. The Journal of Yunnan Administration College, 2019, 21(4): 121-124. ]

[33] 唐晓云. 中国旅游发展政策的历史演进(1949-2013): 一个量化研究的视角 [J]. 旅游学刊, 2014, 29(8): 1527. [Tang Xiaoyun. The evolution of Chinese tourism development policies (1949-2013): A quantitative perspective. Tourism Tribune, 2014, 29(8): 15-27. ]

[34] 韦俊峰, 何瀚林, 明庆忠. 中国休闲农业和乡村旅游政 策的演进特征(2001-2018): 基于政策文本量化分析 [J]. 社会科学家, 2019(3): 84-90. [Wei Junfeng, He Hanlin, Ming Qingzhong. Characteristics of the evolution of Chinese leisure agriculture and rural tourism polices (2001-2018): A quantitative perspective. Social Scientist, 2019(3): 84-90. ]

[35] 刘西涛, 刘璐. 促进乡村旅游发展的政策支持研究 [J]. 现代农业研究, 2018(5): 30-31. [Liu Xitao, Liu Lu. Policy support research on promoting rural tourism development. Modern Agricultural Research, 2018(5): 30-31. ]

[36] Ragin C C. The comparative method: Moving beyond qualitative and quantitative strategies [M]. Berkeley, USA: University of California Press, 1987.

[37] Ragin C C. Fuzzy-set social science [M]. Chicago, USA: University of Chicago Press, 2000.

[38] 伯努瓦・里豪克斯, 查尔斯 $\cdot \mathrm{C}$ ·拉金. QCA 设计原理与 应用 $[\mathrm{M}]$. 杜运周, 李永发, 等译. 北京: 机械工业出版 社, 2017: 25. [Rihoux B, Ragin C C. Configurational comparative method: Qualitative comparative analysis (QCA) and related techniques. Translated by Du Yunzhou, Li Yongfa, et al. Beijing, China: China Machine Press, 2017: 25. ]

[39] Fiss P C. Building better causal theories: A fuzzy set approach to typologies in organization research [J]. Academy of Management Journal, 2011, 54(2): 393-420.

[40] 杨春宇. 中国旅游制度变迁机制及其理论体系构建研 究: 基于新博弯格局视角 [J]. 商业经济与管理, 2011 (12): 76-83. [Yang Chunyu. Chinese tourism evolutionary path analysis and theoretical system construction: Based on the new game pattern view. Journal of Business Economics, 2011(12): 76-83. ]

[41] 梁宏志. 西部民族地区旅游产业集群培育研究: 以云南 省旅游政策变迁为视角 $[\mathrm{J}]$. 云南行政学院学报, 2012, 14(5): 127-131. [Liang Hongzhi. A study on the cultivation of tourism industry clusters in the western ethnic areas: From the perspective of the changes of tourism poli- 
cies in Yunnan Province. The Journal of Yunnan Administration College, 2012, 14(5): 127-131. ]

[42] 丰雷, 蒋妍, 叶剑平, 等. 中国农村土地调整制度变迁中 的农户态度: 基于 1999-2010年 17省份调查的实证分 析 [J]. 管理世界, 2013(7): 44-58. [Feng Lei, Jiang Yan, Ye Jianping, et al. The attitude of farmers in the institutional change of rural land adjustment in China: An empirical analysis based on the survey of 17 provinces from 1999 to 2010. Management World, 2013(7): 44-58. ]

[43] 孙彦斐, 唐晓岗, 刘思源. 乡村文化景观保护的现实境 遇及路径: 基于 “人地关系”的环境教育路径 [J].南京农 业大学学报(社会科学版), 2020, 20(1): 117-126. [Sun Yanfei, Tang Xiaolan, Liu Siyuan. The actual situation and path of rural cultural landscape protection: Environmental education path based on "human- land relationship". Journal of Nanjing Agricultural University (Social Sciences Edition), 2020, 20(1): 117-126. ]

[44] 张薇, 史坤博, 杨永春, 等. 网络與情危机下旅游形象感 知的变化及对出游意向的影响: 以青岛 “天价虾事件” 为例 [J]. 人文地理, 2019, 34(4): 152-160. [Zhang Wei, Shi Kunbo, Yang Yongchun, et al. The change in perceived image and its effect on travel intention with the internet public opinion crisis: A case study of Qingdao's "pricey prawn". Human Geography, 2019, 34(4): 152160. ]

[45] 赵德余, 梁鸿. 政策利益相关者行为模式与规则的渐进 调整: 来自上海医疗卫生改革的经验 [J]. 公共管理学 报, 2009, 6(2): 50-58, 125. [Zhao Deyu, Liang Hong. Behavior model of stakeholder and rules' incremental adjusting during policy process: From Shanghai experi- ence of reconstructing urban' new medical service system. Journal of Public Management, 2009, 6(2): 50-58, 125. ]

[46] 黄震方, 陆林, 苏勤, 等. 新型城镇化背景下的乡村旅游 发展: 理论反思与困境突破 [J]. 地理研究, 2015, 34(8): 1409-1421. [Huang Zhenfang, Lu Lin, Su Qin, et al. Research and development of rural tourism under the background of new urbanization: Theoretical reflection and breakthrough of predicament. Geographical Research, 2015, 34(8): 1409-1421. ]

[47] 易承志. 国家建设、农村土地政策变迁与农民权利保障 [J]. 学术界, 2013(3): 67-75. [Yi Chengzhi. National construction, rural land policy changes and farmers' rights protection. Academics, 2013(3): 67-75. ]

[48] 苏宏元, 黄晓曦. 突发事件中网络谣言的传播机制: 基 于清晰集定性比较分析 [J]. 当代传播, 2018(1): 64-67, 71. [Su Hongyuan, Huang Xiaoxi. The spreading mechanism of internet rumors in emergencies: Based on the clear set qualitative comparative analysis. Contemporary Communication, 2018(1): 64-67, 71. ]

[49] Haandrich L. Charles C. Ragein: Fuzzy-set social science [J]. Politishe Vieteljahressschrift (German Political Science Quarterly), 2000, 41(4): 788-789.

[50] 万笉, 王佃利. 中国邻避冲突结果的影响因素研究: 基 于 40 个案例的模糊集定性比较分析 [J]. 公共管理学 报, 2019, 16(1): 66-76, 172. [Wan Yun, Wang Dianli. Research on the affecting factors of NIMBY conflict outcomes in China: Based on 40 NIMBY conflicts cases through fsQCA. Journal of Public Management, 2019, 16 (1): 66-76, 172. ] 


\title{
Pathway of influence of rural tourism policy change from the perspective of configuration:
}

\section{Qualitative comparative analysis based on Huangshan case study}

\author{
ZHANG Yuangang ${ }^{1}$, WONG IpKin Anthony ${ }^{2 *}$, YU Xiangyang ${ }^{3}$ \\ (1. College of Tourism, Shanghai Normal University, Shanghai 200234, China; \\ 2. School of Tourism Management, Sun Yat-sen University, Guangzhou 510275, China; \\ 3. School of Tourism, Huangshan University, Huangshan 245041, Anhui, China)
}

\begin{abstract}
Under the institutional background of China, the development of rural tourism is closely related to the change of policies. In this study, the dynamic mechanism of rural tourism policy change was explored by using the clear set qualitative comparative analysis method of multivariable interactions. This method emphasizes that through the continuous dialogue of empirical data and relevant theories, the possible causality of the research target can be mapped out from small and medium sample data. This method was used due to the following considerations: First, the main feature of the clear set qualitative comparative analysis method is its treatment of dichotomous variables, especially phenomena "occurring" and "not occurring", thus it is suitable for examining whether there is a change in policy in this study. Second, the core of the qualitative comparative analysis method of clear set is not to consider each condition variable as an independent variable, but to consider it in combination with other variables in different ways. The results of rural tourism policy change in Huangshan depend on different influencing factors at the same time and the use of clear set qualitative comparative analysis method can discover multiple pathways with equivalent results. Third, the relationship between the single factors causing Huangshan rural tourism policy change is not symmetrical and the clear set qualitative comparative analysis method is useful for such analysis. Fourth, the use of qualitative comparative analysis method of clear set can find out multiple pathways with equivalent results. This method can compare different cases in the same area, explore the combination of antecedent conditions in accordance with the theoretical logic, and establish the causal relationship between various combinations of conditions and the interpreted results. Based on the above, this research explored the configuration of the impact of rural tourism policy change, which is composed of organization/leadership change, rural planning policy change, local cultural policy change, host resident participation, and tourism market public opinion. The results show that: 1) Among the individual factors, the change of organization/leadership has a greater influence on the tourism policies at the level of tourism bureau and the participation of host residents has a greater influence on the policies at the city level, but all individual factors are not sufficient for the change of rural tourism policies. 2) Among the 32 alternative path combinations, there are four specific combination pathways with sufficient conditions that affect the changes of rural tourism policies at the municipal level, and there are three specific combination pathways with sufficient conditions that affect the changes of rural tourism policies at the tourism bureau level. 3) For the change of tourism policies at the municipal level, the change of rural planning policy, public opinion of tourism market, and the participation of host residents have similar degrees of influence, and the influence of leadership change of the tourism department is relatively small. 4) For the change of policies at the level of tourism bureau, the change of organization and leadership, the change of rural planning policy, and the change of cultural policy have similar degrees of influence, and host resident participation and public opinion of tourism market have less influence in this pathway.
\end{abstract}

Keywords: rural tourism policy; policy change; pathway of influence; qualitative comparative analysis; Huangshan 